Abstract M31 Table 1 Summary of safety profile at the prespecified interim analysis

\begin{tabular}{|l|c|c|c|c|c}
\hline & $\begin{array}{c}\text { Patients with at } \\
\text { least one } \\
\text { TEAE* }^{*}\end{array}$ & $\begin{array}{c}\text { Patients with at } \\
\text { least one TEAE } \\
\text { related to } \\
\text { pirfenidone } \\
\text { only } \\
\mathbf{n}=63\end{array}$ & $\begin{array}{c}\text { Patients with at } \\
\text { least one TEAE } \\
\text { related to } \\
\text { nintedanib only } \\
\mathbf{n}(\%)\end{array}$ & $\begin{array}{c}\text { Patients with at } \\
\text { least one TEAE } \\
\text { relateds to both } \\
\text { pirfenidone and } \\
\text { nintedanib } \\
\mathbf{n}(\%)\end{array}$ \\
\hline All treatment-related TEAEs (total of 330 events)
\end{tabular}

"Each of the patients could have experienced $\geq 1$ treatment-related event, with the potential for

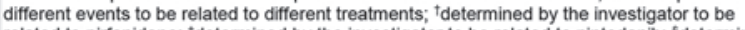
related to pirfenidone; "determined by the investigator to be related to nintedanib; 'sdetermined by the investigator to be related to both pirfenidone and nintedanib

TEAE, treatment-emergent adverse event

of $\geq 1602 \mathrm{mg} /$ day pirfenidone for $\geq 28$ days. Investigators recorded treatment-emergent adverse events (TEAEs), attributing them to pirfenidone, nintedanib, both or neither. Change from baseline FVC, DLco and King's Brief Interstitial Lung Disease (KBILD) score were assessed at 24 weeks. The study is monitored by a data monitoring committee.

Results Eighty-nine patients were enrolled.

A pre-specified interim analysis was conducted once 63 patients (mean age 68.7 years, $85.7 \%$ male) completed $(n=50)$ or discontinued $(n=13) 24$ weeks' combination treatment. Fifty patients had 330 treatment-related TEAEs (Table); 11 patients discontinued due to TEAEs. Two patients had serious treatment-related TEAEs (Table) but none led to death. Final Results for all 89 patients, including change from baseline FVC, DLco and K-BILD score, will be presented at BTS.

Conclusions Combined pirfenidone and nintedanib use for 24 weeks did not reveal a different safety profile to that expected for either treatment alone. Patients had tolerated a stable dose of pirfenidone before initiation of nintedanib, which may explain why investigators attributed more TEAEs to nintedanib than pirfenidone.

Funding F. Hoffmann-La Roche, Ltd./Genentech, Inc.

\section{M32 EFFECT OF PIRFENIDONE ON ALL-CAUSE MORTALITY (ACM) AND FORCED VITAL CAPACITY (FVC) IN IDIOPATHIC PULMONARY FIBROSIS (IPF) PATIENTS WITH LOW FVC AND/OR LOW DLCO: ANALYSIS OF POOLED DATA FROM ASCEND AND CAPACITY}

${ }^{1} \mathrm{SD}$ Nathan, ${ }^{2} \mathrm{U}$ Costabel, ${ }^{3} \mathrm{C}$ Albera, ${ }^{4} \mathrm{KU}$ Kirchgaessler, ${ }^{5} \mathrm{~W}$ Chou, ${ }^{6} \mathrm{PW}$ Noble. ${ }^{1}$ Inova Fairfax Hospital, Falls Church, US; ${ }^{2}$ Ruhrlandklinik, University of Duisburg-Essen, Essen, Germany; ${ }^{3}$ University of Turin, Turin, Italy; ${ }^{4}$ F. Hoffman-La Roche Ltd., Basel, Switzerland; ${ }^{5}$ Genentech Inc., South San Francisco, US; ${ }^{6}$ Cedars-Sinai Medical Centre, Los Angeles, US
Introduction The pivotal trials of the two approved therapies in IPF, pirfenidone and nintedanib, assessed patients with protocol-defined mild to moderate disease. The effect of pirfenidone in patients with more severe lung function impairment warrants further investigation. Pooled Results from ASCEND and CAPACITY studies (NCT01366209, NCT00287729 and NCT00287716) showed a significant reduction at 12 months in the risk of ACM (hazard ratio [HR], 0.52; 95\% CI, 0.31, $0.87)^{1}$ and in decline of percent predicted FVC (\%FVC; $14.8 \%$ vs. $26.3 \%$ patients with $\geq 10 \%$ decline in $\% \mathrm{FVC}$ or death, $\mathrm{p}<0.0001)^{2}$ for patients treated with pirfenidone vs. placebo. We present pooled subgroup analyses from ASCEND and CAPACITY for patients with low baseline\%FVC $(<50 \%)$ and/or low percent predicted diffusing capacity for carbon monoxide $\left(\% \mathrm{DL}_{\mathrm{CO}}<35 \%\right)$ to further inform on treatment effect of pirfenidone in patients with more severe lung function impairment.

Methods ACM was compared using the log-rank test, and HR was estimated using Cox regression. The categorical change in $\%$ FVC was summarised with the percent of patients with $a \geq 10 \%$ absolute decline or death, and treatment comparison was performed using the rank ANCOVA method. Annual rate of FVC decline was estimated using the mixed-effects model.

Results 170 patients (90 pirfenidone, 80 placebo) had low $\%$ $\mathrm{DL}_{\mathrm{CO}}(\mathrm{n}=157)$ or\%FVC $(\mathrm{n}=13)$ at baseline. Treatment with pirfenidone was associated with a $72 \%$ reduction in risk of ACM over 12 months vs. placebo (4 vs. 12 deaths; HR, $0.28 ; 95 \%$ CI, 0.09, 0.86; p=0.018; Table). There was a $56 \%$ relative reduction in the proportion of patients with a ${ }^{3} 10 \%$ absolute decline in $\%$ FVC or death at 12 months vs. placebo $(18.9 \%$ vs. $42.5 \%$; $=0.0038)$. The annual rates of FVC decline were 150 and $278 \mathrm{~mL}$ in the pirfenidone and placebo arms, respectively $(\mathrm{p}=0.003)$.

Conclusions Treatment with pirfenidone resulted in clinically meaningful benefits for ACM and FVC decline in patients with baseline $\%$ FVC $<50 \%$ and/or $\% D_{\mathrm{CO}}<35 \%$. These data suggest that patients with more severe lung function impairment can also benefit from pirfenidone therapy.

\section{REFERENCES}

1. King TE Jr, et al. N Engl J Med 2014;370:2083-2092.

2. Noble PW, et al. Eur Resp J 2016;47:27-30.

Abstract M32 Table 1 ACM and FVC outcomes at 12 months in patients with IPF with low FVC and/or low DLCO at baseline*

\begin{tabular}{lll}
\hline Endpoint at 12 Months & $\begin{array}{l}\text { Patients With Low FVC and/or } \\
\text { Low } \mathrm{DL}_{\mathrm{co}} \\
(\mathbf{n}=170)\end{array}$ & $\begin{array}{l}\text { All Patients } \\
(\mathrm{n}=1247)\end{array}$ \\
\hline $\begin{array}{l}\text { Pirfenidone vs placebo } \\
\text { ACM: HR (95\% Cl) }\end{array}$ & $0.28(0.09-0.86)$ & $0.52(0.31-$ \\
$\begin{array}{l}\text { Pirfenidone vs. placebo: } \\
\geq 10 \% \text { absolute decline in\%FVC or }\end{array}$ & $18.9 \%$ vs. $42.5 \%$ & $0.87)$ \\
death & 150 vs. 278 & $14.8 \%$ vs. \\
Pirfenidone vs. placebo: & $(p=0.003)$ & $26.3 \%$ \\
Annual rate of FVC decline, $\mathrm{mL}$ & $(\mathrm{p}<0.0001)$ \\
\hline
\end{tabular}

*All pirfenidone-treated patients included in these analyses were randomised to receive $2403 \mathrm{mg} /$ day. 